\title{
Electrochemical Push-Pull Scanner with Mass Spectrometry Detection
}

\author{
Dmitry Momotenko, ${ }^{\dagger}$ Liang Qiao, ${ }^{\dagger}$ Fernando Cortés-Salazar, ${ }^{\dagger}$ Andreas Lesch, ${ }^{\ddagger}$ Gunther Wittstock, ${ }^{\ddagger}$ \\ and Hubert H. Girault*, ${ }^{\dagger}$

\begin{abstract}
${ }^{\dagger}$ Laboratoire d’Electrochimie Physique et Analytique, Ecole Polytechnique Fédérale de Lausanne, Station 6, CH-1015 Lausanne, Switzerland

${ }^{*}$ Department of Pure and Applied Chemistry, Faculty of Mathematics and Natural Sciences, Carl von Ossietzky University of Oldenburg, D-26111 Oldenburg, Germany
\end{abstract}

\section{Supporting Information}

ABSTRACT: This manuscript presents a push-pull electrochemical scanner able to image reactivity of initially dry surfaces by scanning electrochemical microscopy (SECM) and to probe molecules present or generated at the surface by mass spectrometry (MS). The proof-of-concept is demonstrated by coupling SECM with matrix-assisted laser desorption/ionization mass spectrometry (MALDI-MS) for imaging latent human fingerprints, which had been in contact with picric acid used here as a model explosive. The push-pull electrochemical scanner has also been coupled with electrospray ionization mass spectrometry (ESI-MS) to assay the activity of surface spotted enzymes. These experimental studies are complemented by 3D finite element simulations solving NavierStokes and diffusion-convection differential equations to optimize the coupling between SECM imaging and mass spectrometry detection.

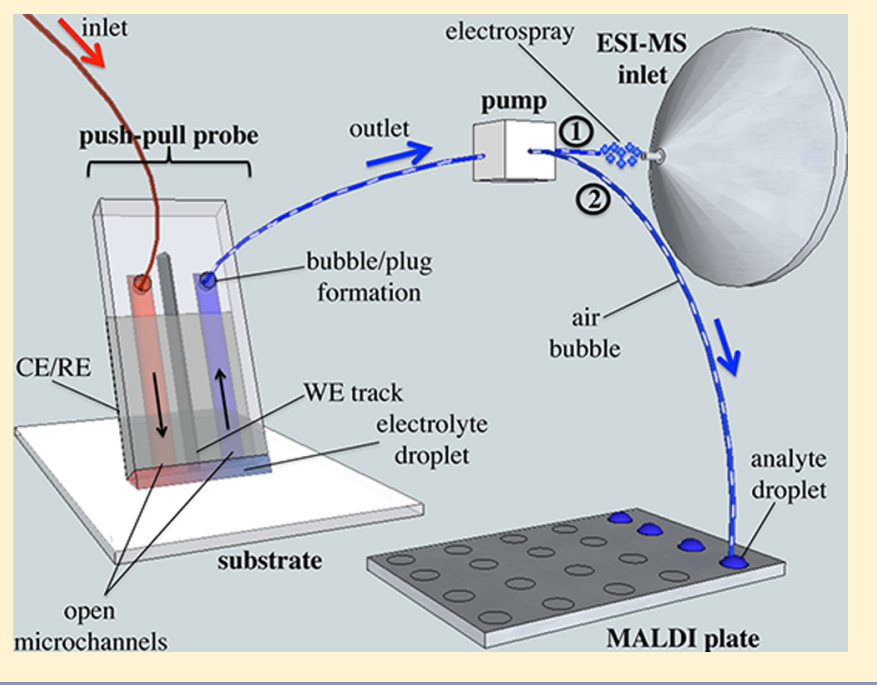

techniques able to control the vertical probe position. These techniques are based on the detection of shear-forces, ${ }^{19-21}$ tip impedance measurements, ${ }^{22,23}$ ion currents in scanning ion conductance microscopy, ${ }^{11,24}$ damping of a vertically modulated microelectrode, ${ }^{25}$ or using combined SECM-AFM probes. $^{26-28}$ Another strategy for keeping $d$ constant was realized within the concept of soft probes for $\mathrm{SECM}^{29-32}$ where the substrate is scanned in contact mode, i.e., when the probe is being brought into soft mechanical contact, bent, and dragged over the substrate in a brushing-like way following the sample topography. Experimental problems when scanning curved, tilted, corrugated, and large substrates approaching a square centimeter can be therefore overcome in this manner, allowing the extension of the SECM scope from proof-ofconcept applications and research tasks to real world problems. ${ }^{33}$ The use of thin polymeric sheets as main probe material provides a certain flexibility and excellent mechanical stability to the probe body. For instance, a tip crash with the substrate, which is often undesired for fragile glass-fabricated UMEs, becomes a very unlikely scenario for soft probes.

Received: April 16, 2012

Accepted: July 4, 2012

Published: July 4, 2012 
The other limitation of SECM for local interfacial analysis lies in the nature of the probe response. By definition, SECM is restricted to the detection of redox active species that could be oxidized or reduced at the UME and/or at the sample. Therefore, SECM is not able to reveal the whole map of chemical and/or electrochemical processes taking place at or near the interface, as the detection of electrochemically inactive species is often troublesome. A possible solution is to measure spatially resolved molecular signals rather than electrochemical response of the probe that could be implemented, for instance, by mass spectrometry (MS) detection methods. For example, scanning mass spectrometry with integrated constant distance positioning ${ }^{34,35}$ was used for visualization of Pt-based catalyst activity for ethane hydrogenation reaction and methanol oxidation. The key element of the probe consisted of two coaxial capillaries, the outer one for feeding reactants to the sample surface and the inner one for pumping off the product mixture to a quadrupole MS-detector. The argon ion current originating from externally fed argon diffusing into the confined space between the precisely positioned capillaries and the sample surface was used for distance control, providing the sample topography, while the relative concentration of product revealed catalytic activity of a catalyst spot.

A similar approach for mapping the spatial electrochemical activity of $\mathrm{Cr} / \mathrm{Pt}$ electrodes was used in scanning capillary microscopy/mass spectrometry technique, ${ }^{36}$ where a coaxial capillary set connected to an electrospray ionization massspectrometer (ESI-MS) was employed for probing electrochemical oxidation of dimethyl-p-phenylenediamine occurring at the working substrate electrode. Successful spatiotemporal experiments with micrometer-scale resolution demonstrated the proof-of-concept; however, the complicated electrochemical cell design would probably limit the application scope of the technique. Recently, Laskin et al. ${ }^{37}$ demonstrated an approach for tissue imaging using nanospray desorption electrospray ionization mass spectrometry. This setup uses minute amounts of solvent confined between two capillaries comprising the probe and the solid analyte for controlled desorption of molecules present on the substrate followed by ionization through self-aspirating nanospray. However, the use of microfluidics provokes another limitation, as the transfer of ultrasmall liquid volumes from sample to the detection unit is affected by mixing inside the liquid flow. In order to retain spatiotemporal information, the liquid sample could be divided into small portions, preventing mutual mixing. This microfluidic approach was used in a droplet-based "chemistrode" 38 for stimulation, recording and analysis of spatiotemporally resolved molecular signals. The authors were able to study the response of living cells to various stimulants (e.g., $\mathrm{Ca}^{2+}$ ions, glucose) with high temporal and spatial resolution by matrixassisted laser desorption ionization mass spectrometry (MALDI-MS), fluorescence microscopy, and fluorescent correlation spectroscopy. However, the need for an additional microfluidic system for the generation of plug arrays and the limitation for the study of hydrophilic surfaces put additional constraints for the use of this technique. Additionally, as the probe was not mounted on a positioning system, the droplet behavior was not studied during probe translation and no scanning was demonstrated even though the probe exhibited spatial resolution at a micrometer scale in a static mode.

The aim of the present work is to combine the high resolving power of SECM for characterizing local (electro)chemical reactivity with the sensitivity and flexibility of MS-detection.
Recently, we integrated microfluidic systems into soft probes to perform SECM experiments over initially, dry horizontal, tilted, or even vertical substrates. Microfluidic fountain pen ${ }^{39}$ and push-pull ${ }^{40}$ probes demonstrated the possibility to avoid immersing the sample under an electrolyte solution that could be a critical condition for delicate samples and to circumvent solvent evaporation typically encountered during SECM imaging in microenvironments. Additionally, the push-pull probe is equipped with two microfluidic channels maintaining a nanoliter droplet at the probe tip by pushing and pulling electrolyte solution to and away from the substrate. The amperometric measurements are performed at the probe tip where the working microelectrode (WE) and the counter/ reference electrode $(\mathrm{CE} / \mathrm{RE})$ are in contact with the nanoliter droplet of electrolyte solution. Herein, we have prepared microfluidic soft SECM probes for simultaneously recording the electrochemical surface reactivity and detecting surface molecules using MALDI-MS and ESI-MS. First, we have coupled SECM imaging of latent human fingerprints with offline MALDI-MS identification of explosive molecules present on a human fingerprint. Second, we have coupled SECM with ESI-MS to characterize the reactivity of surface immobilized enzymes. The integrated microfluidics drives the extracted analyte in plugs separated by air bubbles into the detector preventing the loss of spatiotemporal resolution. Numerical 3D modeling of the microfluidic push-pull probe demonstrated the influence of convective fluxes on measured amperometric signal and revealed the capabilities of the microfluidic platform for characterization of surface reactivity in chemical and electrochemical mode when scanning in contact regime.

\section{EXPERIMENTAL SECTION}

Chemicals. Ferrocene methanol $\left(\mathrm{FcCH}_{2} \mathrm{OH}, \geq 97 \%\right.$, Sigma-Aldrich), $\mathrm{KNO}_{3}$ ( $\geq 99 \%$, Buchs, Switzerland), 4-aminophenyl phosphate monosodium salt hydrate ( $p$-aminophenyl phosphate, PAPP, from Biosynth), $\mathrm{NH}_{4} \mathrm{HCO}_{3}(\geq 97 \%$, Fluka), L-lysine monohydrate ( $\geq 98 \%$, Fluka), and 2,4,6-trinitrophenol (picric acid, Fluka) were used as received, as well as methanol (HPLC grade, AppliChem) and acetic acid ( $\geq 99.5 \%$, Fluka). Deionized water was produced by a Milli-Q plus 185 model from Millipore (Zug, Switzerland). Push-pull probes were produced using $100 \mu \mathrm{m}$ thick polyethylene terephthalate, Melinex (PET, Dupont, Wilmington, DE, USA), and $50 \mu \mathrm{m}$ polyethylene/polyethylene terephthalate (PE/PET, Payne, Wildmere Road, Banbury, England) lamination foils. Lasermachined tracks in PET were filled by Electrador carbon ink (Electra Polymer \& Chemicals Ltd., Roughway Mill, Dunk Green, England) for the $\mathrm{WE}$ and by $\mathrm{Ag} / \mathrm{AgCl}$ ink (ERCON, Wareham, MA, USA) for the CE/RE electrode.

Push-Pull Probe Fabrication. The fabrication of microfluidic push-pull probes was done as previously described. ${ }^{40}$ In brief, the tracks for open microchannels of the microfluidic system, working electrode, and counter/reference electrode were ablated in a PET film through metallic masks using a 193 nm ArF excimer laser beam (Lambda Physik, Göttingen, Germany, fluence $=0.35 \mathrm{~J}$, frequency $=50 \mathrm{~Hz})$. After ablation, open microchannels (60 $\mu \mathrm{m}$ width, $40 \mu \mathrm{m}$ depth, and 6.3-6.8 $\mathrm{cm}$ length) were laminated with PE/PET lamination foil, whereas a uniform Parylene $\mathrm{C}$ coating of defined thickness (1$10 \mu \mathrm{m}$ ) was applied over the WE side after filling the respective microchannel (55 $\mu \mathrm{m}$ width, $30 \mu \mathrm{m}$ depth, and $4 \mathrm{~cm}$ length) with carbon ink and after a curing step at $80{ }^{\circ} \mathrm{C}$ for $1 \mathrm{~h}$. A crosssection of the probe was then exposed by mechanical cutting 


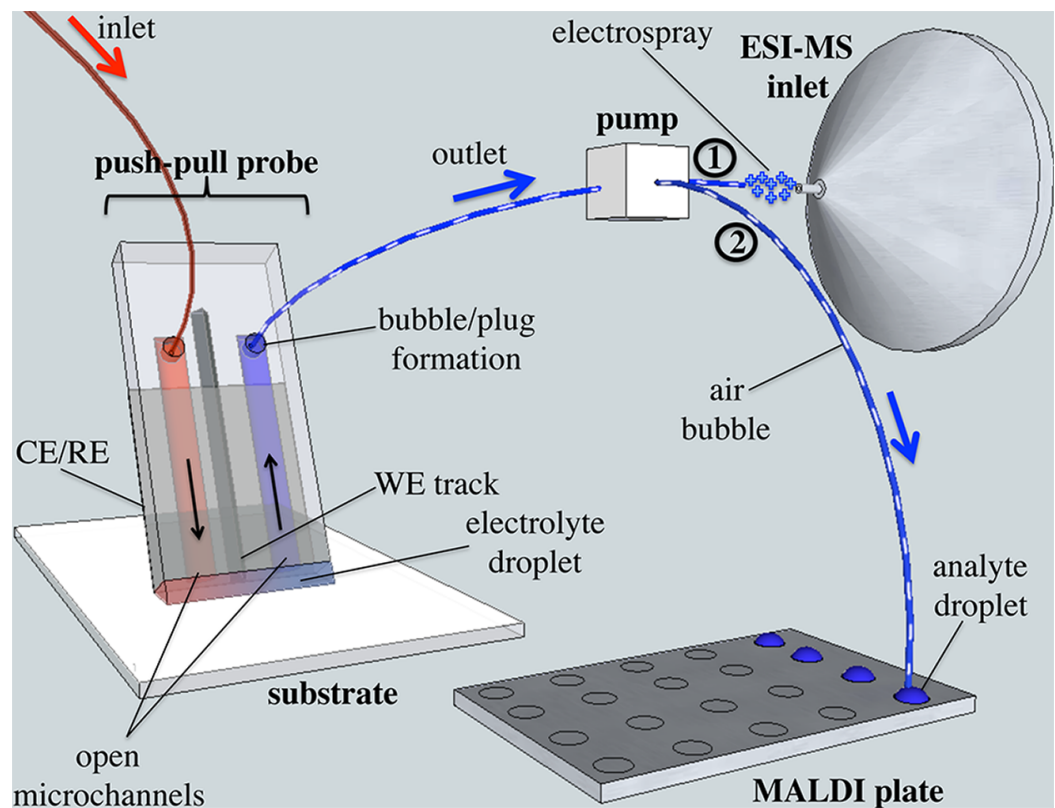

Figure 1. Schematic view of the general operation principle of the push-pull probe. The liquid is pumped via the microchannels of the probe connected to external pumps through microcapillaries (liquid flow directions are indicated with arrows). Electrochemical measurements are carried out within a nanoliter droplet of electrolyte using $\mathrm{WE}$ and $\mathrm{CE} / \mathrm{RE}$ integrated in the probe operating in contact mode over a substrate; chemical detection of analyte plugs separated by air bubbles is performed using either (1) ESI-MS or (2) MALDI-MS tools. The mutual mixing of analyte is prevented by generating air bubbles in between extracted analyte portions at the junction between the probe and external capillary connected to the pumping unit.

with a scalpel blade. The quality of the electrodes and the shape of exposed area were inspected with a scanning laser microscope (VK 8700, Keyence).

The probe was connected to an external pumping system (syringe pump KD Scientific, 250 and $500 \mu \mathrm{L}$ Gastight syringes 1700 series, Hamilton and/or peristaltic pump IPC-N 24, Ismatec, equipped with Tygon tubing with internal diameter (ID) of $250 \mu \mathrm{m}$ ) using fused silica capillaries with $150 \mu \mathrm{m}$ ID. A custom-built probe holder with predefined inclination angle of $70^{\circ}$ allowed reliable microfluidic connection via reservoirs (N-124S, Nanoport Assembly, Upchurch Scientific) and simplified probe-sample alignment using a custom-built worm gear.

SECM Measurements. SECM measurements were carried out using a custom-built SECM setup controlled by SECMx software using lift-off routine ${ }^{30}$ and comprising an IVIUM compactstat (IVIUM Technologies, The Netherlands) operating in a classical three-electrode mode. Data analyses were carried out offline using MIRA software. ${ }^{30,41}$ All potentials are reported with respect to the $\mathrm{Ag} / \mathrm{AgCl}$ integrated quasireference electrode. All the samples were mounted on the bottom of a flat cell construction and investigated at room temperature $\left(20 \pm 2{ }^{\circ} \mathrm{C}\right)$.

Chemical Detection of Contaminants on a Human Fingerprint. All fingerprints were artificially contaminated with various amounts of picric acid (2,4,6-trinitrophenol). Typically, the finger of a volunteer was contacted with a drop $(0.1-2 \mu \mathrm{L})$ of $28.4 \mathrm{mM}$ aqueous solution of contaminant, and after drying under the gentle flux of nitrogen, the fingerprint was left on a gold substrate $(100 \mathrm{~nm}$ Au layer deposited on glass using typical physical vapor deposition protocols). Caution: picric acid is sensitive to shocks and friction when dry. Always keep it dissolved in water. Additionally, picric acid is toxic if adsorbed through skin and can cause skin irritation. Afterward, a latent fingerprint was developed by the cyanoacrylate fuming technique as described elsewhere. ${ }^{42}$ The substrate with a fingerprint was inserted for a short time $(0.5-2 \mathrm{~min})$ inside a small (100-300 mL volume) closed chamber mounted on a heater containing a few $\mu \mathrm{L}$ of cyanoacrylate glue (Loctite 406 from Henkel) and a small open flask with warm water (30-40 ${ }^{\circ} \mathrm{C}$ ) providing a certain humidity level in the chamber atmosphere.

The dry developed fingerprint was scanned in SECM feedback mode with a push-pull probe monitoring the current of $\mathrm{FcCH}_{2} \mathrm{OH}$ oxidation at $0.4 \mathrm{~V}$. Typically, the applied microfluidic flow rates for pumping electrolyte $(2 \mathrm{mM}$ $\mathrm{FcCH}_{2} \mathrm{OH}, 40 \mathrm{mM} \mathrm{KNO}{ }_{3}$ ) varied in the range of $6-40 \mu \mathrm{L} /$ $\mathrm{h}$ due to instability of the droplet size that is based on instrumental pumping limitations. During SECM scanning, the liquid aspirated from the push-pull probe was collected and a small amount of this liquid fraction $(1-2 \mu \mathrm{L})$ was deposited on a MALDI target plate and analyzed on a Bruker Microflex timeof-flight mass spectrometer in positive reflectron mode.

Chemical/Electrochemical Characterization of Immobilized Enzyme Activity. The immobilization of alkaline phosphatase (from native calf, AbD Serotec, $4000 \mathrm{U} / \mathrm{mg}$ ) was performed on a highly hydrophobic polyvinylidene difluoride (PVDF) membrane taking advantage of its high binding affinity for proteins. A PVDF membrane was prewetted by methanol and water. Afterward, $0.5-1 \mu \mathrm{L}$ of an aqueous, $1 \mathrm{mg} / \mathrm{mL}$ enzyme solution was deposited on the membrane. After drying, the lateral scanning was performed under a thick layer of deionized water and by continuously pumping an aqueous solution containing $11 \mathrm{mM}$ PAPP, $1 \mathrm{mM}$ lysine, and $20 \mathrm{mM}$ $\mathrm{NH}_{4} \mathrm{HCO}_{3}$ through the inlet microfluidic channel to the probe tip at $0.4 \mu \mathrm{L} \cdot \mathrm{min}^{-1}$ and monitoring the probe current at $0.35 \mathrm{~V}$ for the electrochemical detection of PAP whose production is catalyzed by the enzyme. The reaction volume was pulled through the outlet channel, and this aspirated liquid was divided into a sequence of plugs separated by air bubbles, 
collected in a capillary, and then driven to an ESI-MS detection unit. The detection unit contained a polyimide microchip emitter for ESI that was described previously ${ }^{43}$ and a Thermo LTQ velos mass-spectrometer. Analyte collected inside the capillary was infused into the microchip emitter via a microchannel $(50 \mu \mathrm{m} \times 100 \mu \mathrm{m})$ integrated into the microchip at a flow rate of $20 \mu \mathrm{L} / \mathrm{h}^{-1}$. A high voltage was applied to a microelectrode integrated in the device and in contact with the microchannel for ESI. An ESI buffer of 50\% water, $49 \%$ methanol, and $1 \%$ acetic acid was infused via another microchannel at a flow rate of $40 \mu \mathrm{L} / \mathrm{h}^{-1}$. The two flows mixed just before arriving at the microelectrode; therefore, the microelectrode was always in contact with acidic medium leading to a relatively stable ESI, regardless of the air bubbles being introduced along with analyte. PAP and lysine were both protonated and detected under a positive MS mode. During ESI, the microchip emitter was placed just in front of the MS inlet $(0.8 \mathrm{~cm}$ away). A positive high voltage of $3.7 \mathrm{kV}$ was applied on the emitter, and the MS was grounded. No sheath gas was used to help ESI. The mass spectrometer was scanning under a normal scan rate for a range of mass-to-charge ratio $\mathrm{m}$ / $z$ of 50-500. All the mass spectrometer conditions were kept constant during experiments for good quantification of the analytes (more details in Supporting Information SI-1).

Numerical Simulations. The numerical modeling of a push-pull probe was built using commercially available finite element software COMSOL Multiphysics (version 3.5a) running on Mac Pro with four $2.66 \mathrm{GHz}$ central processing units and $9.8 \mathrm{~Gb}$ of RAM. Steady-state numerical analysis of Navier-Stokes and diffusion/convection equations was performed in a three-dimensional computational domain. The convergence was reached by solving these partial differential equations (PDEs) sequentially, i.e., computing diffusion/ convection PDEs on top of the solution of Navier-stokes equation as the fluid's properties are assumed to not be affected by the change in concentration of dissolved species. For more details of a FEM simulation procedure and geometrical parameters of the model and mesh, see Supporting Information SI-2.

\section{RESULTS AND DISCUSSION}

Push-Pull Principle. The push-pull probe can be divided into two modules: an electrochemical cell for amperometric measurements and a microfludic unit. The former consists of an UME as WE probing surface electrochemical reactivity and a relatively large $\mathrm{CE} / \mathrm{RE}$ used to control the applied voltage (Figure 1). An important requirement for the probe design is to bring the WE as close as possible to the substrate in order to decrease $d$ (Figure 2) and to introduce the CE/RE in the vicinity of the WE to avoid instability of the amperometric signal caused by droplet size variations. Here, the gap between WE and CE/RE was determined by the thickness of the Parylene $\mathrm{C}$ coating while in contact mode $d$ was kept at a micrometer and submicrometer distance from the substrate providing sensitive SECM detection. ${ }^{40}$

The microfluidic unit of the probe incorporates two open microchannels connected to an external syringe pumping system that drives an electrolyte solution to and away from the substrate. When an electrolyte solution is pumped into the microfluidic channel, a constantly renewed nanoliter droplet is formed at the probe tip as the excess of liquid is aspirated into the other microfluidic channel and driven to further MS analysis. The major constraint stemming from the use of
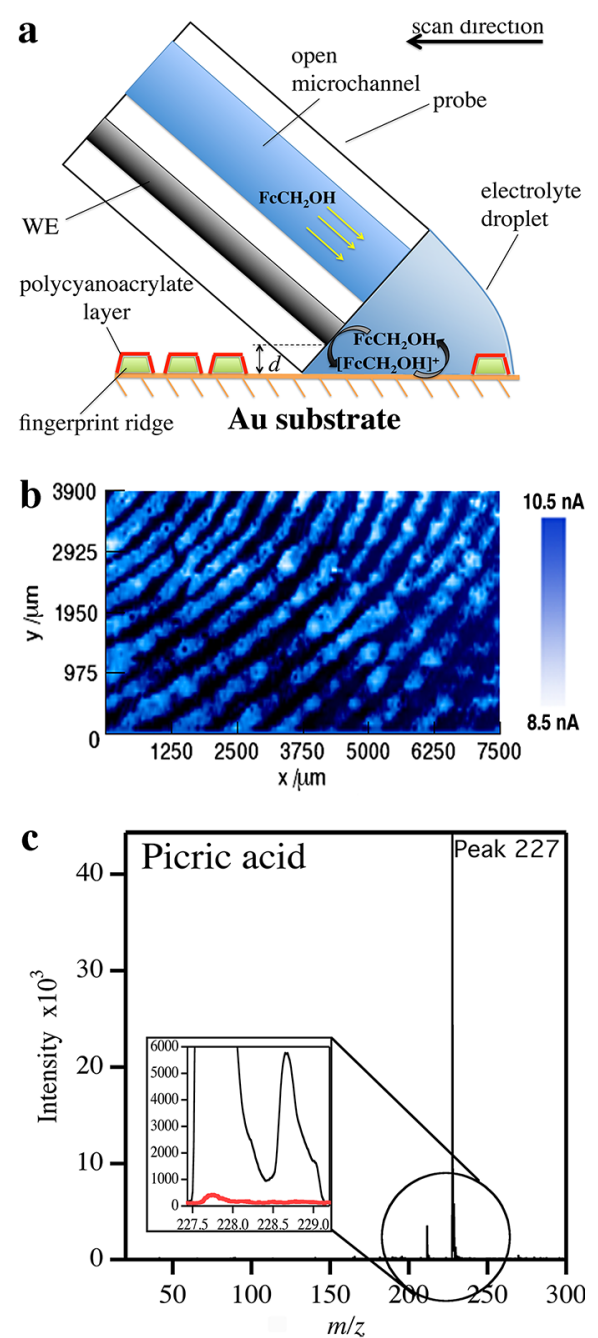

Figure 2. Fingerprint imaging with the push-pull scanner. (a) The concept implemented for fingerprint detection with push-pull probes (not to scale). (b) SECM image $\left(E_{\mathrm{T}}=0.4 \mathrm{~V}\right.$, step size of $25 \mu \mathrm{m}$ in $x$ and $75 \mu \mathrm{m}$ in $y$ directions, translation rate $v=300 \mu \mathrm{m} \mathrm{s}^{-1}$ and $75 \mu \mathrm{m}$ $\mathrm{s}^{-1}$, respectively) of a latent human fingerprint and (c) MALDI-MS spectra of the collected liquid portions. The inset on the graph reveals the magnified view on the selected area, and the red line indicates a MALDI-MS spectrum of the analyte collected from a fingerprint (28.5 nmol of picric acid initially deposited on a finger).

microfluidics is the mixing process in the laminar flow leading to a loss of spatiotemporal resolution. Mixing is taking place most likely due to the parabolic velocity profile across the microfluidic channel leading to a fast dispersion of analyte concentration (details in Supporting Information SI-3). According to numerical modeling of a liquid flow inside a $100 \mu \mathrm{m}$ ID capillary, convective-dominated mixing could result in a complete loss of spatial resolution between two analyte plugs within a few $\mathrm{cm}$ of a flow path even at moderate liquid flow rates. The push-pull probe overcomes this limitation in two conceivable ways: either by setting the ratio of pushing and pulling rates in such a way that air bubbles are pumped in jointly with the liquid sample when the push-pull probe operates over an initially dry substrate or by introducing a controllable gap between capillary and the probe hence introducing a small leak to the junction between the capillary and the probe holder. The latter strategy is an efficient way of forming a plug sequence at a given frequency and is preferable 
for push-pull experiments under a thick solution layer or in a solution bulk. Hence, the liquid portions collected at the sample surface are driven into the MS detector without loss of spatial resolution and could be analyzed online with ESI-MS or divided into small portions for MALDI detection. The pushpull scanner is therefore advantageous as it does not require the use of additional microfluidic devices (e.g., based on a Tjunction ${ }^{44}$ ) for plug sequence generation.

Fingerprint Analysis. Detection, visualization, and enhancement of latent human fingermarks are important tasks in forensic sciences. Besides traditional fingerprint detection methods, a broad range of chemical and physical visualization techniques are available nowadays, taking advantage of nanoparticle deposition for improving optical detection sensitivity, ${ }^{45,46}$ spectroscopic methods such as FT-IR imaging, ${ }^{47}$ and scanning probe techniques (Kelvin probe). ${ }^{48}$ However, fingerprint identification often needs to be complemented with chemical analysis of trace evidence, for instance by the use of Raman spectroscopy, ${ }^{49,50}$ ATR-FT-IR technique ${ }^{51}$ for simultaneous fingermark detection coupled with chemical analysis, ion chromatography of gunshot residue, ${ }^{52}$ or by measuring local electrochemical currents in surface plasmon resonance. ${ }^{53}$ Recently, fingerprint identification was performed by SECM in a feedback mode based on detection of silver nanoparticles ${ }^{54}$ or benzoquinone-tagged proteins. ${ }^{31}$ Chemical detection of contaminants using these two methods could be intricate, as the development of a fingermark involves numerous immersing and/or washing steps. In this work, we demonstrate simultaneous SECM imaging of a human fingerprint on a conductive substrate and MS-detection of an explosive contaminant, i.e., picric acid, using a cyanoacrylate fuming development protocol.

The scheme of fingermark analysis using the SECM feedback mode is represented in Figure 2a. Due to a very high affinity of cyanoacrylate to fingerprint residue, the impressions from friction ridges are typically covered with a thin layer of insulating polycyanoacrylate, while the rest of the fingerprint remains untreated. Therefore, when the probe was scanned over the substrate, the variations of amperometric signal were attributed to $\mathrm{FcCH}_{2} \mathrm{OH}$ recycling at the conductive $\mathrm{Au}$ substrate (when probe passed uncovered areas) and blocking the redox mediator mass-transport when the WE was over fingerprint ridges. The resulting positive and negative feedback currents plotted as a function of tip coordinate resulted in a contrasted image of a fingerprint, like the one shown in Figure 2b. Cyanoacrylate fuming technique was implemented on conductive substrates and hence is complementary to the existing nanoparticle and benzoquinone tagging methods for fingerprint imaging with SECM, as the latter ones could only be used on inert nonconductive surfaces. Moreover, silver stained fingerprints could drastically lose contrast after amperometric detection as positive SECM feedback is based on silver nanoparticle dissolution. ${ }^{55}$ In contrast, cyanoacrylate fumed fingerprints are chemically and mechanically stable and could be scanned repeatedly with push-pull probe. Effortless protocol for fingerprint development makes cyanoacrylate fuming an easy-to-use method for SECM detection.

Additional difficulties in SECM imaging could appear if the fingerprint-containing substrate does not present sufficient chemical stability under ambient conditions. For instance, we have observed a destruction of a copper substrate $(40 \mathrm{~nm} \mathrm{Cu}$ layer deposited on glass) placed under electrolyte $(2 \mathrm{mM}$ $\mathrm{FCCH}_{2} \mathrm{OH}$ and $40 \mathrm{mM} \mathrm{KNO}_{3}$ ) solution after ca. $1 \mathrm{~h}$
(Supporting Information SI-4). Most likely, this could be attributed to oxidation of a copper layer by dissolved oxygen. However, no changes related to copper oxidation or dissolution were observed with push-pull operating over initially dry fingerprint samples demonstrating an additional asset of the push-pull scanner. Hence, the present scanner provides a platform that could be used for SECM imaging over delicate or chemically unstable substrates.

In addition to SECM imaging, MALDI analysis of the liquid portions collected from the scanned fingerprint were performed showing the possibility to detect down to $28.5 \mathrm{nmol}$ of explosive deposited on the volunteer's finger (Figure 2c). One has to note that the transferred amount of picric acid could significantly vary depending on the type of substrate used and the transferred amount from a finger, since most of this contaminant is retained on the skin of the finger. ${ }^{56}$ Typically, MS detection methods are capable of detecting nanogram amounts of explosives on a human skin or finger; ${ }^{57}$ hence, push-pull fingerprint analysis demonstrates fair detection limits. Most likely, relatively low efficiency of the push-pull approach could arise from a set of limiting factors affecting the sensitivity of chemical detection on fingerprints using the cyanoacrylate strategy: (a) the degree of development, as the polymer layer blocks the analyte deposited on a fingerprint, (b) the size of scanning area, and (c) fluidic flow rates due to dilution of analyte. Therefore, moderate development time and slow microfluidic rates are preferable for push-pull chemical analysis and should bring sensitivity of the push-pull detection to a higher level under optimized conditions.

SECM/ESI-MS Characterization of Immobilized Enzyme Reactivity. One of the key features of the microfluidic push-pull scanner is the ability to stimulate, control, and readout chemical/electrochemical events occurring at the interface. Figure $3 a$, and $3 b$ demonstrate this concept showing the operation of a push-pull probe over the substrate covered with a thick layer of deionized water. The delivery of a proper stimulation through microfluidic unit of the probe establishes a high local concentration of chemical stimulants just at the probe tip, where the WE senses an electrochemically active species. The chemical composition of the local environment at the interface can be simultaneously analyzed by driving the analyte into the ESI-MS detection unit through the microfluidic unit.

Here, we aim at imaging a spot of enzymes immobilized on a surface to model an immunoassay. To this end, we observed the conversion of PAPP molecules into the electrochemically active $p$-aminophenol (PAP) occurring at immobilized alkaline phosphatase (ALP). An important advantage of the push-pull scanning approach is the possibility to modify locally an environment without significantly altering the bulk properties of the solution: for instance, we could deliver the substrate PAPP along with lysine in a $\mathrm{NH}_{4} \mathrm{HCO}_{3}$ buffer solution with $\mathrm{pH}$ of 8.0 providing proper conditions for the enzymatic reaction, as ALP is most reactive in a $\mathrm{pH}$ range from 8 to 10 . Lysine worked as an internal standard with constant concentration in the microfluidic block for MS quantification of PAP since the two compounds have very close isoelectric point and molecular weight giving similar ionization efficiency under ESI conditions. Figure $3 c$ shows the corresponding SECM and ESI-MS data from a single line scan over an ALP spot. The ratio between peak intensities of PAP $\left(I_{\mathrm{PAP}}\right)$ and lysine $\left(I_{\text {Lys }}\right)$ from ESI-MS was calculated to identify the amount of PAP generated from different areas of the PVDF membrane with immobilized ALP. 

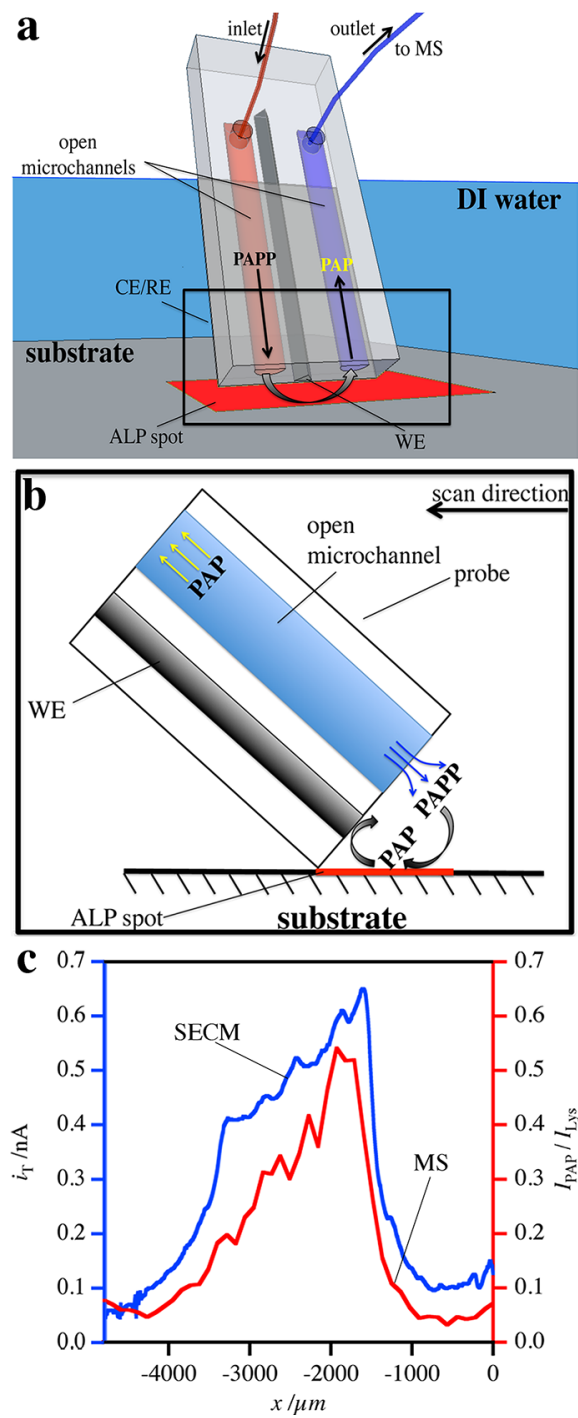

Figure 3. Characterization of enzyme activity with push-pull probes. (a) Three dimensional and (b) magnified view on the selected area in lateral projection schematically representing the push-pull strategy for simultaneous SECM and MS detection of a product of enzymatic reaction: the stimulant (PAPP) is delivered via microfluidics of the probe and is converted at the immobilized enzyme (ALP) to product (PAP), which is sensed at the WE and is pulled into an aspiration microfluidic channel for further MS-identification. (c) Experimental $\operatorname{SECM}\left(E_{\mathrm{T}}=0.35 \mathrm{~V}\right.$, step size of $10 \mu \mathrm{m}$, translation rate $v=50 \mu \mathrm{m} \mathrm{s}^{-1}$, depicted in blue) and corresponding ESI-MS (axis on the right, shown in red) line scans over an alkaline phosphatase spot. The ESI-MS intensity of PAP $I_{\mathrm{PAP}}$ is represented as relative to the ion current of lysine $I_{\text {Lys }}$ used as internal standard.

As can be seen, amperometric and MS measurements are well correlated proving the capabilities of the push-pull scanning approach for parallel chemical and electrochemical activity characterization, although there is an offset between ESI-MS and SECM data (vide infra). Without doubt, the success of chemical detection during such push-pull experiments can be limited by a number of factors significantly affecting the results. For example, high salt concentrations, the instability of MS signal due to air bubbles within the liquid flow, and the adsorption of analyte on the capillary or the microfluidic unit of the probe could obstruct chemical detection. The use of a sheath buffer introduced in the microchip emitter to stabilize the ESI partially overcame these limitations.

Numerical Simulations of Push-Pull Chemical/Electrochemical Imaging. The resolution power of chemical/ electrochemical detection in a push-pull scanning mode is difficult to estimate experimentally due to the relatively high error value for correlation between MS and SECM data. In contrast, numerical simulations could provide an extensive description of the whole system and give access to various parameters, i.e., yielding fluid velocity fields, concentrations, and mass-fluxes of species.

Figure $4 \mathrm{a}$ illustrates the convection field surrounding the push-pull probe. As expected, fluid velocity reaches significantly high values in the order of $1 \mathrm{~mm} \cdot \mathrm{s}^{-1}$ near the probe tip forming spherical-like profiles near open microfluidic channels. Most likely, the collection of an analyte from the substrate occurs in this highly convective region close to the aspirating microchannel. Typically, convective fluxes are very undesired for SECM experiments limiting the resolution of the technique since convection disturbs the diffuse layer structure formed at the UME. ${ }^{58}$ In the case of the push-pull probe, the use of microfluidics establishes the convection-dominated mass-transport at the probe tip, however, not in the microscopic confinement in between the WE and the substrate (inset in Figure 4a). As it can be seen, in this case, the fluid movement is locally suppressed due to arising friction forces, and therefore, the diffusion layer between SECM probe and substrate remains almost unperturbed allowing amperometric detection. This result provides a theoretical evidence for the suppression of convective contribution to the overall current within the microdomain formed at the probe tip as it was proposed previously ${ }^{39,40}$ and at the same time reveals the advantages of contact mode arrangement that overcomes difficulties in SECM imaging experiments even under convection-dominated conditions.

Figure $4 b, c$ depicts the simulated line scans in $x$ and $y$ directions performed with a push-pull probe over a $1 \mathrm{~mm}^{2}$ squared substrate where the electrochemically inert stimulant molecules delivered by the microfluidic unit are converted to the product detected amperometrically at the UME and transferred into the aspirating microchannel (similar to the experiment described in the previous section). Of course, there is a remarkable asymmetry between chemical and electrochemical response in push-pull experiments, most likely due to the arrangement of a contact scanning mode of a soft probe and asymmetric probe design. This effect is conspicuous particularly in the high frequency axis scans (along $x$ direction, Figure $4 b$ ), where the data of chemical detection lag behind the SECM signals. Surprisingly, the asymmetry of performance along the $y$ axis is relatively small despite the $\mathrm{WE}$ and aspirating channel are dislocated with respect to each other. The simulated chemical detection exhibits a lack of lateral resolution compared to SECM data from integrated probes. This fact is most likely observed, as the diffusion layer of micro-WE is smaller than the convective layer at the aspirating microfluidic channel (as could be seen from Figure 4a) determining the spatial confinement of MS detection. An additional consequence of a contact mode arrangement for soft probes is a slight discrepancy of SECM measurements with chemical detection data along the high frequency axis leading to an inaccurate measurement of the reactive pattern position at the interface (Figure $4 \mathrm{~b}$ ). This result provides a theoretical support to the experimental observations during parallel SECM/ESI-MS characterization of 


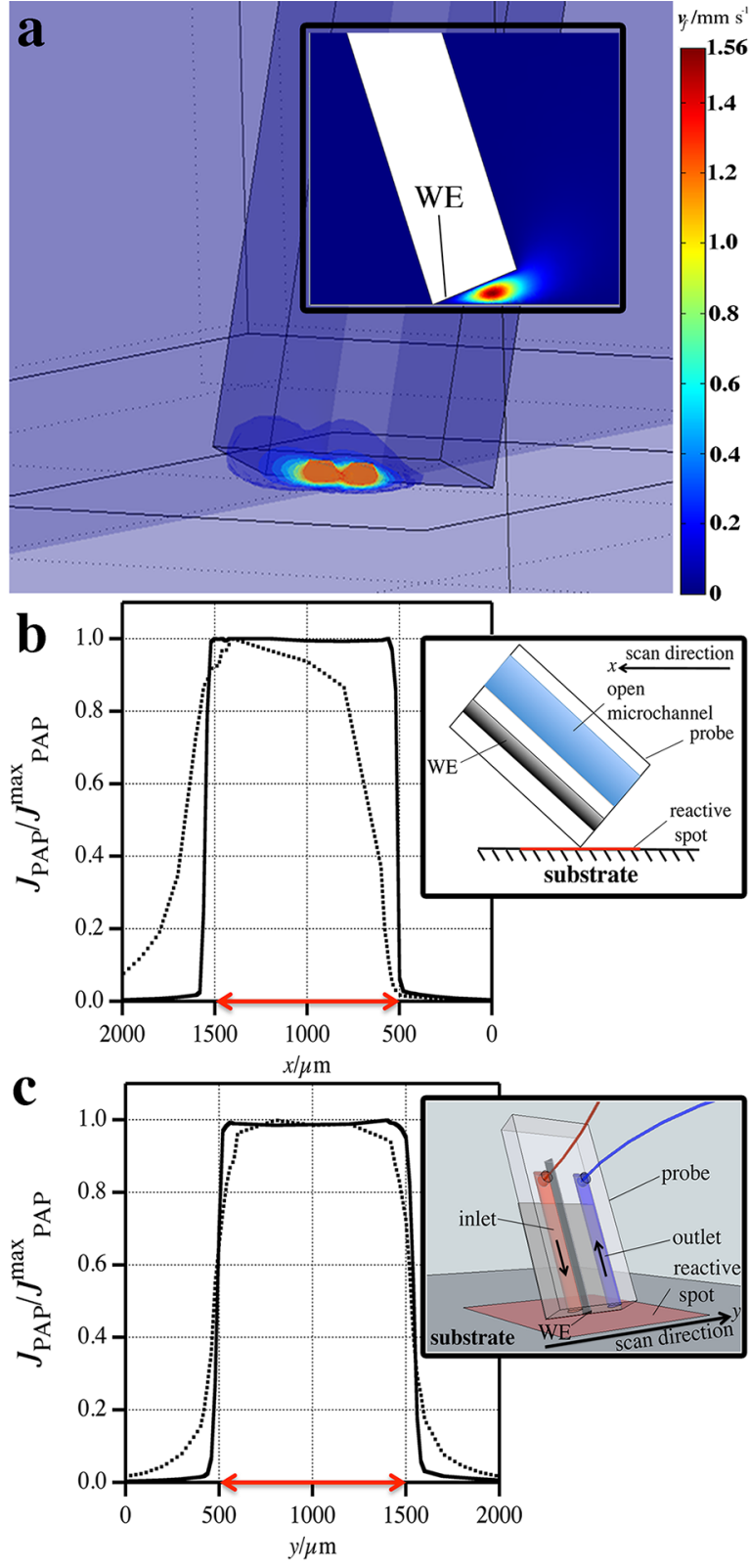

Figure 4. (a) Simulated fluid velocity fields $\left(v_{\mathrm{f}}\right.$, in $\mathrm{mm} \mathrm{s}^{-1}$, see colorbar on the right) surrounding push-pull probe operating under an electrolyte layer. Boundary condition for inlet sets flow rate to $20 \mu \mathrm{L}$ $\mathrm{h}^{-1}$ and pressure boundary condition at the outlet. The inset demonstrates velocity field in the lateral projection of the medial cross-section. For clarity, the position of the WE is indicated. (b, c) The simulated SECM (solid) and MS (dotted) line scans across the reactive spot $\left(1 \mathrm{~mm}^{2}\right.$ square, kinetic rate constant toward delivered stimulant $\left.k=1 \times 10^{-3} \mathrm{~m} \mathrm{~s}^{-1}\right)$ in $x$ and $y$ directions are represented as a relative value (for convenience of representation, normalized to maxima of the flux or electrical current). The location of the reactive spot is shown with a red double arrow bar. Schematic representations on the graph insets clarify the probe and reactive spot positions with respect to each other and demonstrate the implemented scanning direction in each case.

reactivity spot described in the previous section (Figure 3c). As can be seen, the experimental chemical/electrochemical data from line scans are in qualitative agreement with the numerical result. This effect of mismatching chemical detection data with actual position of the pattern seems to be a typical consequence of the use of microfluidics and was reported previously. ${ }^{59}$

\section{CONCLUSION}

The present study demonstrates a novel approach for control and analysis of chemical phenomena occurring at interfaces with high spatial resolution accomplished with sensitive and versatile MS detection. The electrochemical push-pull scanner combines the resolving power of SECM with sensitive and versatile mass-spectrometric detection tools providing chemical identification to electrochemical events. This strategy incorporates the advantages of soft probes for SECM and microfluidics allowing electrochemical measurements within a nanodroplet of electrolyte over initially dry substrates or under a thick solution layer. Operating with a small amount of liquid, the push-pull probe initiates and reads out interfacial chemical events by measuring electrochemical and molecular responses. As proof of concept, various analytical applications were achieved, such as latent human fingerprint imaging accompanied with chemical detection of contamination by explosives and parallel chemical/ electrochemical characterization of immobilized enzyme activity. Three dimensional finite element simulations rationalized the operational capabilities of simultaneous SECM and MS detection with a push-pull platform for characterization of surface reactivity.

\section{ASSOCIATED CONTENT}

\section{S Supporting Information}

Details of ESI experiments, FEM simulations including mesh, boundary conditions, and constant parameters, the simulated convection-diffusion mixing process in a capillary and the example of push-pull scanning experiments over delicate samples as well as COMSOL report files are available free of charge via the Internet at http://pubs.acs.org.

\section{AUTHOR INFORMATION}

\section{Corresponding Author}

*E-mail: hubert.girault@epfl.ch. Telephone: +41-21-693 3145. Fax: +41-21-693 3667.

\section{Notes}

The authors declare no competing financial interest.

\section{ACKNOWLEDGMENTS}

This work was supported by SNCF Grants 20735001 and 20628506 and by a collaborative grant of SNF and Deutsche Forschungsgemeinschaft (SNF 20PA21_121570/1; DFG Wi $1617 / 10)$ "High throughput SECM ${ }^{-}$imaging". We also acknowledge Valerie Devaud and Cyrille Hibert for technical support and Peter Pechy for kindly supplying the picric acid employed in this work.

\section{REFERENCES}

(1) Engstrom, R. C.; Weber, M.; Wunder, D. J.; Burgess, R.; Winquist, S. Anal. Chem. 1986, 58, 844-848.

(2) Bard, A. J.; Fan, F. R. F.; Kwak, J.; Lev, O. Anal. Chem. 1989, 61, $132-138$.

(3) Bard, A. J.; Fan, F. R. F.; Pierce, D. T.; Unwin, P. R.; Wipf, D. O.; Zhou, F. Science 1991, 254, 68-74.

(4) Souto, R. M.; Gonzalez-Garcia, Y.; Battistel, D.; Daniele, S. Corros. Sci. 2012, 55, 401-406.

(5) Guadagnini, L.; Chiavari, C.; Martini, C.; Bernardi, E.; Morselli, L.; Tonelli, D. Electrochim. Acta 2011, 56, 6598-6606. 
(6) Tefashe, U. M.; Nonomura, K.; Vlachopoulos, N.; Hagfeldt, A.; Wittstock, G. J. Phys. Chem. C 2012, 116, 4316-4323.

(7) Tefashe, U. M.; Loewenstein, T.; Miura, H.; Schlettwein, D.; Wittstock, G. J. Electroanal. Chem. 2010, 650, 24-30.

(8) Ye, H.; Lee, J.; Jang, J. S.; Bard, A. J. J. Phys. Chem. C 2010, 114, $13322-13328$.

(9) Chen, X. X.; Eckhard, K.; Zhou, M.; Bron, M.; Schuhmann, W. Anal. Chem. 2009, 81, 7597-7603.

(10) Sun, P.; Laforge, F. O.; Abeyweera, T. P.; Rotenberg, S. A.; Carpino, J.; Mirkin, M. V. Proc. Natl. Acad. Sci. U. S. A. 2008, 105, 443-448.

(11) Takahashi, Y.; Shevchuk, A. I.; Novak, P.; Murakami, Y.; Shiku, H.; Korchev, Y. E.; Matsue, T. J. Am. Chem. Soc. 2010, 132, 1011810126.

(12) Liu, X. H.; Ramsey, M. M.; Chen, X. L.; Koley, D.; Whiteley, M.; Bard, A. J. Proc. Natl. Acad. Sci. U. S. A. 2011, 108, 2668-2673.

(13) Takahashi, Y.; Miyamoto, T.; Shiku, H.; Ino, K.; Yasukawa, T.; Asano, R.; Kumagai, I.; Matsue, T. Phys. Chem. Chem. Phys. 2011, 13, 16569-16573.

(14) Laforge, F. O.; Velmurugan, J.; Wang, Y. X.; Mirkin, M. V. Anal. Chem. 2009, 81, 3143-3150.

(15) Xiong, H.; Kim, J.; Kim, E.; Amemiya, S. J. Electroanal. Chem. 2009, 629, 78-86.

(16) Danieli, T.; Colleran, J.; Mandler, D. Phys. Chem. Chem. Phys. 2011, 13, 20345-20353.

(17) Grisotto, F.; Metaye, R.; Jousselme, B.; Geffroy, B.; Palacin, S.; Charlier, J. J. Mater. Chem. 2011, 21, 15962-15968.

(18) Cornut, R.; Nunige, S.; Lefrou, C.; Kanoufi, F. Electrochim. Acta 2011, 56, 10701-10707.

(19) Nebel, M.; Eckhard, K.; Erichsen, T.; Schulte, A.; Schuhmann, W. Anal. Chem. 2010, 82, 7842-7848.

(20) Takahashi, Y.; Shiku, H.; Murata, T.; Yasukawa, T.; Matsue, T. Anal. Chem. 2009, 81, 9674-9681.

(21) Yamada, H.; Ogata, M.; Koike, T. Langmuir 2006, 22, 79237927.

(22) Kurulugama, R. T.; Wipf, D. O.; Takacs, S. A.; Pongmayteegul, S.; Garris, P. A.; Baur, J. E. Anal. Chem. 2005, 77, 1111-1117.

(23) Alpuche-Aviles, M. A.; Wipf, D. O. Anal. Chem. 2001, 73, 48734881.

(24) Comstock, D. J.; Elam, J. W.; Pellin, M. J.; Hersam, M. C. Anal. Chem. 2010, 82, 1270-1276.

(25) McKelvey, K.; Snowden, M. E.; Peruffo, M.; Unwin, P. R. Anal. Chem. 2011, 83, 6447-6454.

(26) Derylo, M. A.; Morton, K. C.; Baker, L. A. Langmuir 2011, 27, 13925-13930.

(27) Leonhardt, K.; Avdic, A.; Lugstein, A.; Pobelov, I.; Wandlowski, T.; Wu, M.; Gollas, B.; Denuault, G. Anal. Chem. 2011, 83, 29712977.

(28) Smirnov, W.; Kriele, A.; Hoffmann, R.; Sillero, E.; Hees, J.; Williams, O. A.; Yang, N. J.; Kranz, C.; Nebel, C. E. Anal. Chem. 2011, 83, 4936-4941.

(29) Cortes-Salazar, F.; Träuble, M.; Li, F.; Busnel, J. M.; Gassner, A. L.; Hojeij, M.; Wittstock, G.; Girault, H. H. Anal. Chem. 2009, 81, 6889-6896.

(30) Cortes-Salazar, F.; Momotenko, D.; Lesch, A.; Wittstock, G.; Girault, H. H. Anal. Chem. 2010, 82, 10037-10044.

(31) Cortes-Salazar, F.; Momotenko, D.; Girault, H. H.; Lesch, A.; Wittstock, G. Anal. Chem. 2011, 83, 1493-1499.

(32) Lesch, A.; Momotenko, D.; Cortes-Salazar, F.; Wirth, I.; Tefashe, U. M.; Meiners, F.; Vaske, B.; Girault, H. H.; Wittstock, G. J. Electroanal. Chem. 2012, 666, 52-61.

(33) Mirkin, M. V.; Nogala, W.; Velmurugan, J.; Wang, Y. X. Phys. Chem. Chem. Phys. 2011, 13, 21196-21212.

(34) Li, N.; Assmann, J.; Schuhmann, W.; Muhler, M. Anal. Chem. 2007, 79, 5674-5681.

(35) Li, N.; Eckhard, K.; Assmann, J.; Hagen, V.; Otto, H.; Chen, X. X.; Schuhmann, W.; Muhler, M. Rev. Sci. Instrum. 2006, 770841021084102-7.
(36) Modestov, A. D.; Srebnik, S.; Lev, O.; Gun, J. Anal. Chem. 2001, $73,4229-4240$

(37) Laskin, J.; Heath, B. S.; Roach, P. J.; Cazares, L.; Semmes, O. J. Anal. Chem. 2012, 84, 141-148.

(38) Chen, D.; Du, W. B.; Liu, Y.; Liu, W. S.; Kuznetsov, A.; Mendez, F. E.; Philipson, L. H.; Ismagilov, R. F. Proc. Natl. Acad. Sci. U. S. A. 2008, 105, 16843-16848.

(39) Cortes-Salazar, F.; Lesch, A.; Momotenko, D.; Busnel, J. M.; Wittstock, G.; Girault, H. H. Anal. Methods 2010, 2, 817-823.

(40) Momotenko, D.; Cortes-Salazar, F.; Lesch, A.; Wittstock, G.; Girault, H. H. Anal. Chem. 2011, 83, 5275-5282.

(41) Wittstock, G.; Asmus, T.; Wilhelm, T. Fresenius' J. Anal. Chem. 2000, 367, 346-351.

(42) Lee, H. C.; Gaensslen, R. E. Advances in fingerprint technology; CRC Press: Boca Raton, Florida, USA; 1994.

(43) Qiao, L.; Lu, Y.; Liu, B. H.; Girault, H. H. J. Am. Chem. Soc. 2011, 133, 19823-19831.

(44) Garstecki, P.; Fuerstman, M. J.; Stone, H. A.; Whitesides, G. M. Lab Chip 2006, 6, 693-693.

(45) Becue, A.; Champod, C.; Margot, P. Forensic Sci. Int. 2007, 168, $169-176$.

(46) Spindler, X.; Hofstetter, O.; McDonagh, A. M.; Roux, C.; Lennard, C. Chem. Commun. (Cambridge, U. K.) 2011, 47, 5602-5604.

(47) Crane, N. J.; Bartick, E. G.; Perlman, R. S.; Huffman, S. J. Forensic Sci. 2007, 52, 48-53.

(48) Williams, G.; McMurray, N. Forensic Sci. Int. 2007, 167, 102109

(49) Day, J. S.; Edwards, H. G. M.; Dobrowski, S. A.; Voice, A. M. Spectrochim. Acta A 2004, 60, 563-568.

(50) Day, J. S.; Edwards, H. G. M.; Dobrowski, S. A.; Voice, A. M. Spectrochim. Acta A 2004, 60, 1725-1730.

(51) Ricci, C.; Bleay, S.; Kazarian, S. G. Anal. Chem. 2007, 79, 57715776.

(52) Gilchrist, E.; Smith, N.; Barron, L. Analyst 2012, 137, 15761583.

(53) Shan, X. N.; Patel, U.; Wang, S. P.; Iglesias, R.; Tao, N. J. Science 2010, 327, 1363-1366.

(54) Zhang, M. Q.; Girault, H. H. Electrochem. Commun. 2007, 9, $1778-1782$.

(55) Zhang, M. Q.; Wittstock, G.; Shao, Y. H.; Girault, H. H. Anal. Chem. 2007, 79, 4833-4839.

(56) Cotte-Rodriguez, I.; Takats, Z.; Talaty, N.; Chen, H. W.; Cooks, R. G. Anal. Chem. 2005, 77, 6755-6764.

(57) Justes, D. R.; Talaty, N.; Cotte-Rodriguez, I.; Cooks, R. G. Chem. Commun. (Cambridge, U. K.) 2007, 2142-2144.

(58) Combellas, C.; Fermigier, M.; Fuchs, A.; Kanoufi, F. Anal. Chem. 2005, 77, 7966-7975.

(59) Kottke, P. A.; Degertekin, F. L.; Fedorov, A. G. Anal. Chem. 2010, 82, 19-22. 